\title{
Criminologie
}

\section{L'abandon de la carrière criminelle : quelques témoignages}

\section{Pierre Pinsonneault}

Volume 18, numéro 2, 1985

Le vol à main armée à Montréal

URI : https://id.erudit.org/iderudit/017218ar

DOI : https://doi.org/10.7202/017218ar

Aller au sommaire du numéro

Éditeur(s)

Les Presses de l'Université de Montréal

ISSN

0316-0041 (imprimé)

1492-1367 (numérique)

Découvrir la revue

\section{Citer cet article}

Pinsonneault, P. (1985). L'abandon de la carrière criminelle : quelques témoignages. Criminologie, 18(2), 85-116. https://doi.org/10.7202/017218ar

\section{Résumé de l'article}

This article summarizes some of the findings of research done on the relinquishing of a criminal career. It is based on about twenty interviews, carried out between July and November 1984, with multirecedivist ex-criminals who had abandoned their criminal careers for at least five years prior to the study. We isolated the factors that dissuaded them from continuing this way of life as well as others that caused them to adopt a more conventional lifestyle.

It is a question of the difficulties connected with a criminal way of life whether it be with one's criminal colleagues, rival gangs, informers, or the police, lawyers and the courts. There follows a series of bad experiences concerning prison life : the accumulation of sentences and years of prison, the living conditions, problems with the officers and guards, the presence of other inmates. But there are also positive things one discovers in prison whether it be new knowledge, new responsibilities or the help of people outside, such as Alcoholics Anonymous or the chaplains or at the emotional level, of a wife or mate.

We see, based on these factors, some of the difficulties that the ex-criminal must face if he wants to persevere in following a conventional lifestyle.
Ce document est protégé par la loi sur le droit d'auteur. L'utilisation des services d’Érudit (y compris la reproduction) est assujettie à sa politique d'utilisation que vous pouvez consulter en ligne.

https://apropos.erudit.org/fr/usagers/politique-dutilisation/ 


\section{L'ABANDON DE LA CARRIÈRE CRIMINELLE :}

QUELQUES TÉMOIGNAGES Pierre Pinsonneault*

This article summarizes some of the findings of research done on the relinquishing of a criminal career. It is based on about twenty interviews, carried out between July and November 1984, with multirecedivist excriminals who had abandoned their criminal careers for at least five years prior to the study. We isolated the factors that dissuaded them from continuing this way of life as well as others that caused them to adopt a more conventional lifestyle.

It is a question of the difficulties connected with a criminal way of life - whether it be with one's criminal colleagues, rival gangs, informers, or the police, lawyers and the courts. There follows a series of bad experiences concerning prison life : the accumulation of sentences and years of prison, the living conditions, problems with the officers and guards, the presence of other inmates. But there are also positive things one discovers in prison whether it be new knowledge, new responsibilities or the help of people outside, such as Alcoholics Anonymous or the chaplains or at the emotional level, of a wife or mate.

We see, based on these factors, some of the difficulties that the ex-criminal must face if he wants to persevere in following a conventional lifestyle.

L'un des directeurs de service dans une très grosse entreprise est, par le fait même, responsable de centaines d'employés. D'autres occupent des positions aussi diverses que camionneur, comédien, contracteur, inspecteur, mécanicien, professeur ou technicien, enfin un seul est prestataire de l'Aide sociale. Presque tous sont mariés et père de famille et ils sont tous, à tout le moins, dans la trentaine.

Des gens à peine différents de ceux qu'il nous est donné de croiser quotidiennement dans la rue, des gens bien ordinaires à première vue! Ces apparences sont cependant trompeuses, car ce sont tous d'ex-criminels multirécidivistes qui ont, entre autres délits, commis de nombreux vols à main armée, certains ont même

* Chercheur, Centre international de criminologie comparée, Université de Montréal. 
tué et tous ont, en conséquence purgé de nombreuses et de longues peines de prison.

Ils ont maintenant abjuré le crime et cela depuis de nombreuses années. Au moment où nous les avons rencontrés, leur casier judiciaire était en effet sans tache depuis au moins cing ans donc, selon les études consacrées à la récidive ils nous offraient des garanties sérieuses d'avoir abandonné la carrière criminelle.

Mais comment sont-ils devenus ce qu'ils sont aujourd'hui? Quand? Pourquoi? Comment, en fait, ont-ils décidé d'abandonner? Est-ce qu'à un moment donné certains événements, certaines influences, certaines personnes ont joué un rôle dans leur prise de décision? Fut-il facile de prendre une telle décision? Ont-ils eu à faire face à des difficultés particulières pour la maintenir? Autant de questions auxquelles nous nous sommes intéressés dans le cadre d'une recherche consacrée à l'abandon de la carrière criminelle.

Dans les balises qui circonscrivent cet article nous ne pouvions aborder tous ces thèmes de façon intensive, ce qui a été fait ailleurs (Pinsonneault, 1984), c'est pourquoi nous ne mettrons l'accent ici que sur les facteurs constitutifs de la décision d'abandonner une carrière criminelle et de se maintenir dans le $x$ droit chemin». Cet article se présente donc comme un triptyque consacré à l'abandon. Il y a d'une part, les premiers déboires liés au style de vie criminel auxquels s'additionnent les mauvaises expériences reliées à la vie carcérale et, d'autre part, les aspects positifs d'une vie fort différente, telle que la perçoit l'ex-criminel qui envisage un changement d'orientation radical. Le troisième volet, enfin, se consacre aux difficultés inhérentes à persister dans cette décision.

Nos données sont issues de dix-sept entrevues en profondeur au cours desquelles nous avons privilégié l'approche qualitative (entretien non directif) avec d'anciens voleurs à main armée francophones de la région de Montréal. Tous âgés de plus de trente ans, ils avaient fait de nombreux stages en prison, mais présentaient un casier judiciaire vierge depuis au moins cinq ans. Ces rencontres qui se sont échelonnées de juillet à novembre 1984, duraient en moyenne de trois à quatre heures et avaient ordinairement lieu au domicile du sujet ou à un endroit de son choix.

C'est à partir de certaines expériences vécues par ces exdétenus, expériences qu'ils nous ont relatées spontanément et, nous 
le croyons, avec une très grande franchise, que nous avons dressé un tableau essentiellement descriptif d'un certain nombre de facteurs qui ont joué un rôle dans leur décision d'abandonner leurs activités criminelles. On pourrait, bien entendu, s'interroger sur la valeur heuristique de tels témoignages dans le contexte de la recherche en criminologie, ceci n'est pas notre objectif ici, car nous nous sommes limités dans le présent travail, à organiser et à décrire ce qui nous a été relaté.

Dans une étape ultérieure nous pourrions procéder systématiquement à une validation concurrente de nos données mais, nous le répétons, notre but pour le moment, ne se situe pas au-delà du processus descriptif : nous avons, pour utiliser une analogie, photographié des situations et des expériences sans, sinon inintentionnellement porter un jugement sur leur valeur et leur importance relatives dans le processus de l'abandon de la carrière criminelle. Une analyse en ce sens resterait à faire.

Mais avant de dresser ce tableau et des facteurs dissuadant d'une vie axée sur le crime et de ceux incitant à une vie normale, faisons d'abord un rapide tour d'horizon des rares écrits dans le domaine, car très peu de recherches se sont, à ce jour, penchées systématiquement sur le problème de l'abandon de la carrière criminelle (Conklin, 1981, p. vi). On peut cependant dégager quelques constantes de celles qui s'y sont intéressées. Il y a, d'abord, le fait constaté par plusieurs, que la détermination de l'individu à abandonner est le facteur moteur de cette décision (Meisenhelder, 1977, Waller, 1974, Cusson, 1983). En effet, comme le constate parmi d'autres Irwin (1970), l'ex-criminel serait tiraillé entre deux aspects de sa personnalité. Il y a d'un côté son «identité criminelle latente» et de l'autre son désir d'assumer une identité sociale plus conventionnelle.

Cette prise de décision qui consiste à s'installer confortablement dans la normalité se ferait plus tardivement chez le criminel que chez la moyenne des gens, comme l'ont souligné d'une part les Glueck (1974) et d'autre part Cormier et son équipe de McGill dans des travaux échelonnés de 1959 à 1975. En effet, selon leurs recherches, on assiste dans ce cas à un processus de «maturation tardive", qui correspond d'ailleurs, statistiquement, à une diminution de la récidive à partir d'un certain âge (Cormier et al., 1965). À quelques nuances près, ce processus se situerait autour de la trentaine. 
Suite à une série d'expériences désagréables liées à la vie criminelle et carcérale et auxquelles s'ajoute la crainte d'être de nouveau incarcéré (Meisenhelder, 1977, West, 1978, Cusson, 1983), l'individu décide de changer son style de vie : cette décision est éclairée et fait suite à un calcul de "coûts et bénéfices» (Meisenhelder, West, Cormier, et al., Cusson). Comme le signale, entre autres West, le criminel realise qu'il y a de plus en plus d'avantages à retirer de la vie conventionnelle et qu'il y aurait de plus en plus de désagréments à affronter, s'il poursuit dans la voie du crime. Quels sont donc ces avantages et inconvénients?

Les auteurs que nous avons consultés, sauf Cusson et dans une moindre mesure Meisenhelder, se sont peu attardés aux aspects négatifs de la vie carcérale, sinon pour mentionner qu'ils existent. Aucun, et Meisenhelder est particulièrement intransigeant sur ce sujet, n'accorde une importance considérable à l'aide institutionnelle dans la détermination du détenu à abandonner. Même l'aide postinstitutionnelle, elle-même, est. remise en question par les études de Waller.

Par contre, les auteurs, qui se sont penchés sur le problème de l'abandon, sont cependant unanimes à constater qu'une situation professionnelle intéressante et la présence d'une compagne, et le cas échéant d'une famille, sont des facteurs lourds dans la décision d'arrêter et de persévérer dans cette voie. En somme, l'ex-criminel qui acquiert les signes extérieurs du conformisme social serait devenu un citoyen ordinaire. D'autres catalyseurs peuvent aider à prendre et maintenir la décision d'arrêter. Ces précipitants peuvent être : la fréquentation de nouveaux amis (Waller, 1974), le fait de retourner aux études (West, 1978), d'avoir des activités extra-professionnelles ou extra-familiales, comme la pratique des sports ou d'un passe-temps (Irwin, 1970), de contrôler son alcoolisme (Waller, 1974). En somme, comme le soulignait fort laconiquement Cusson (1983), il faut «réussir dans autre chose».

Comme nous avons pu le constater dans les travaux qui se sont intéressés au problème de l'abandon, la dualité dissuasionincitation, comparable à l'adage populaire du bâton et de la carotte, revient fréquemment. La plupart des auteurs cependant accordent plus d'importance aux éléments associés à une vie normale comme facteurs primant dans la décision d'abandonner, négligeant ainsi, quoique à des degrés divers, les souvenirs traumatisants hérités de l'activité criminelle. 
Sans privilégier, ni par le fait même négliger, l'un ou l'autre des membres du couple «bâton-carotte» nous allons, en nous inspirant des entrevues que nous avons réalisées, tenter de voir ce qu'ils recouvrent. Nous décrirons donc, tour à tour, les facteurs dissuadant d'une vie axée sur le crime puis ceux incitant à une vie normale.

\section{A. FACTEURS DISSUADANT D'UNE VIE AXÉE SUR LE CRIME}

Deux facteurs peuvent contribuer à dissuader le criminel de poursuivre une vie axée sur le crime. Il y a d'une part les nombreux ennuis occasionnés par son style de vie et, d'autre part, certaines mauvaises expériences reliées à la vie carcérale.

\section{a) Déboires liés au style de vie criminelle}

Collègues criminels, bandes rivales, informateurs, policiers, avocats et tribunaux peuvent constituer autant de sources de frustration et de découragement pour celui qui s'adonne à une vie de crime.

Les amis criminels deviennent souvent un facteur d'incitation sinon des initiateurs au crime, mais cet effet d'entraînement ne se fait pas ressentir uniquement chez les plus jeunes, il peut également jouer après avoir abandonné pendant un certain temps :

Je rencontre les "gars» au hasard, je me fais embarquer dans un truc d'extorsion, ça m'a coûté 16 mois, avec une femme et un enfant. J'avais décidé (...) de laisser tomber, quand j'avais une job et que je me suis marié. Mais les chums... je suis influençable...

Plus tard, avec ses associés, on peut faire face à des problèmes de répartition du butin, ou encore à des problèmes avec les femmes; problèmes qui souvent sont solutionnés à coups de revolver ou de dénonciations.

Les bandes rivales représentent un danger potentiel considérable au même titre que la police dans le milieu criminel. En fait, il faut se battre sur plusieurs fronts. Un ex-gangster résume bien la situation lorsqu'il dit : "...ça sonne chez toi, tu te demandes si c'est les 'boeufs' ou les gangs rivaux». 
Les dénonciations sont également à redouter dans le milieu criminel, surtout quand on sait que le travail policier en est fortement tributaire. Ainsi, le danger peut surgir à l'improviste : «n'importe qui peut te vendre» affirme un ex-voleur, et même les dénonciations sont à craindre dans le milieu carcéral, comme le soulignait un ex-détenu qui considérait que la présence des stools a joué un certain rôle dans sa décision d'abandonner :

Quand tu vois cette bande de cochons qui vendraient n'importe qui pour n'importe quoi. C'est pas le plus important mais ça a aidé (à décider d'abandonner).

À mener une vie de criminel, on doit évidemment s'attendre à avoir un certain nombre de problèmes avec les forces policières. C'est ainsi que certains décideront de se retirer après s'être «fait tirer dessus» ou encore de s'être fait "passer à tabac» ou encore plus simplement suite à la pression policière. Ce harcèlement, d'ailleurs, ne s'arrête pas suite à l'abandon. Quelqu'un qui a été, pendant sa période criminelle active, associé avec des criminels notoires, dont la plupart sont maintenant décédés, suite à des *accidents de travail», nous a avoué que quatre ans après sa sortie de prison, les policiers l'inquiétaient encore.

Arrêté, le criminel doit faire face au système de justice. C'est maintenant le jeu souvent ardu de la négociation avec des avocats, souvent des avocats marrons, avec des procureurs aux longues dents et des juges qui n'ont pas toujours la mémoire courte. C'est en somme la nécessité d'apprendre à composer avec la justice, alors que déjà à la ligne de départ on est souvent perdant. C'est ainsi que les honoraires d'avocat, les «fix» et les sentences exemplaires constituent des expériences fort désagréables pour le criminel.

D'autant plus que pour le criminel vieillissant, la vie devient de plus en plus difficile, de plus en plus risquée, de moins en moins amusante, de plus en plus stressante et cela jusqu'au jour où enfin il se fait prendre pour la dernière fois.

... en vieillissant, tu deviens de moins en moins brave, de plus en plus nerveux, tu penses aux conséquences plus que quand t'es jeune.

Car le criminel d'habitude réalise que plus il commet de délits plus la probabilité de se faire prendre est élevée et plus le risque d'y rester s'accentue : 
... des vols de banque, c'est bon pour les jeunes! Si tu y penses tu ne le fais pas. Tu sais jamais si tu vas te faire tirer quand tu vas en sortir (de la banque).

Ainsi, tel un cancer, les mauvaises expériences associées à la vie du criminel le rongent lentement. Concurremment aux multiples déboires occasionnés, soit par ses pairs criminels, soit par les agents du système répressif, voilà que souvent il a perdu progressivement confiance en lui-même et en ses capacités. Cependant, les vexations ne sont pas terminées pour autant. Il lui faut, encore une fois, retourner en prison, et ce n'est pas de gaieté de coeur qu'il franchit la porte de sa cellule, car il sait maintenant, suite à ses nombreuses incarcérations antérieures, ce qui l'attend en dedans.

b) Mauvaises expériences reliées à la vie carcérale - Pour les multirécidivistes l'accumulation des sentences et des années d'incarcération entraîne inévitablement un certain écoeurement, car en vieillissant, il devient de plus en plus difficile et pénible de «faire du temps». Un ex-détenu nous raconte sa $\mathrm{X}^{e}$ arrestation et les sentiments qu'il a ressentis à ce moment précis de sa vie :

... là c'était pu un «trip». Je ne m'en fous pu, je me demande quel christ de trip que je fais, je ne voulais pu prendre de "vacances». Ie suis tout «fucké». Je me demande pourquoi j'ai fait cela. Ie commence à être écoeuré du milieu carcéral, où il n'y a même pas moyen d'avoir une conversation intelligente...

Écœuré parce que pour le criminel adulte habitué à la belle vie, malgré certains avatars, à une vie plutôt bohème, les conditions de vie en prison avec ses horaires stricts, l'existence de règlements contraignants et quelquefois appliqués plus ou moins arbitrairement, la surveillance quasi constante exercée par des gardiens ou, ce qui semble pire, par des médias électroniques, les décisions plus ou moins despotiques des gardiens et des autorités du pénitencier, les transferts plus ou moins justifiés d'un pénitencier à l'autre, les différentes formes de rationnements pour des gens qui ne se privaient pas de gaspiller, de dilapider, les conditions d'hygiène à l'époque des cellules à «bucket», la nourriture de la cafétéria, tout cela enfin, et beaucoup d'autres éléments reliés aux structures et au fonctionnement même des institutions carcérales, représentent, en fait, l'antithèse de ce qu'il a vécu jusqu'alors dans les bons moments. Il est donc inévitable qu'il en souffre et qu'il en soit écœuré! 
À cela s'ajoutent un certain nombre de problèmes qui nous apparaissent plus spécifiques à quelques-uns. Il s'agit de certains types de relations avec des agents de gestion de cas ou des gardiens. Ainsi, certains détenus se seraient fait imposer, en prison, l'obligation d'apprendre un métier, alors qu'ils préféraient de beaucoup poursuivre leurs études secondaires, abandonnées lors de leur période délinquante.

On ne voulait pas que j'aille à l'école, ils voulaient que je travaille dans une «shop» de métal. Comme je ne voulais pas y aller, ils m'ont envoyé au trou pendant quatre à cinq jours. Puis après je lisais toute la nuit, pour être inapte à travailler le jour.

Un autre ex-détenu, de son côté, n'a pas vécu le même problème, mais il nous indique comment certains agents du système peuvent être mal perçus par les détenus, même lorsqu'ils, semblet-il, tentent d'aider :

J'ai trouvé «ben tannant» de recommencer toujours à raconter (mon histoire) aux différents officiers de classement. Quand t'es longtemps en dedans t'en passes beaucoup. Personne ne me croyait. Après une demi-heure le criminologue m'a demandé de lui raconter la vraie histoire, je lui ai dit : «t'es pareil comme ceux du $4^{\circ}$, t'es rien qu'un flic». C'est du «stock» quand personne ne te croit.

Les relations avec les gardiens sont plus transparentes : ceux-ci représentent un groupe au départ antipathique aux yeux des prisonniers. Ainsi, manifester une sympathie quelconque vis-àvis d'un gardien, même quelquefois dans les plus petites choses de la vie quotidienne de gens qui, quand même, se côtoient pendant des années, peut conduire un détenu à subir l'ostracisme de ses pairs. On nous explique cette philosophie des relations entre les gardiens et les détenus :

Tu peux pas être correct avec les screw. Si un screw t'offre une cigarette, ou si tu lui offres du feu, les gars vont te dire : «s'il échappe sa cigarette, tu vas la ramasser pour lui!» C'est pour cela qu'il faut aller contre tous les règlements, sinon ils (détenus) vont penser que tu es du côté de l'administration.

Si en somme, les relations entre détenus et gardiens ne se situent, généralement pas au niveau de l'amitié, il en va quelquefois de même dans les relations entre les détenus eux-mêmes. La crainte d'avoir une rixe avec les durs en dedans, peut chez certains occuper un espace important dans leur décision d'arrêter : 
J'avais des problèmes de bataille en dedans, toujours avec les mêmes gars, à chaque fois que j'y retournais. Je ne voudrais pas y retourner.

Mais c'est souvent une image négative moins brutale qui contribue à inciter le détenu à envisager l'abandon. Ainsi, l'exemple de détenus qui reviennent constamment en prison ou encore qui purgent de très longues peines d'emprisonnement, par l'exemple déplorable qu'ils incarnent, peut inciter un détenu à abandonner, de peur de se retrouver dans une situation similaire un jour.

Voici comment réagissait l'un deux face à d'autres détenus qui revenaient toujours :

On dirait qu'il y en a qui cherchent leur sécurité en dedans. On dirait que la prison c'est un siphon qui te ramène en dedans, le gars ne comprend pas pourquoi il retourne en dedans.

Un autre, pour sa part a été frappé par des détenus qui purgeaient de lourdes peines :

Moi, j'ai sept ans. Tu vois des gars qui en ont pour vingt ans. Il y a des gars qui te disent : «prends ton mal en patience, tu en as que pour sept ans, moi j'en ai pour vingt». Il y en a, par contre, qui en ont moins que toi. Tu te dis ça pourrait m'arriver vingt ans, me retrouver en dedans pour une «secousse".

L'âge est un facteur important dans la décision d'abandonner la carrière criminelle car, avec le temps, le criminel d'habitude, devient de plus en plus stressé au moment de passer à l'acte. L'usure occasionnée par les retours successifs en prison se fait de plus en plus ressentir et il se sent de moins en moins «capable de faire du temps». D'autre part, l'écœeurement occasionné par les mauvaises expériences liées à la vie criminelle devient de plus en plus insoutenable. Il apparaît, alors que ces accumulations de déboires cascadent vers une saturation qui contribue à procéder à une remise en question.

C'est fort souvent à l'approche de la trentaine que le détenu prend conscience du fait qu'il doit prendre une décision importante : ou bien il continue dans la voie qu'il a jusqu'alors suivie et ce, en pesant les conséquences éventuelles (risque de se faire tuer, blesser, lourde peine la prochaine fois, la «perpette», etc.), ou il se recycle. Pour la majorité de ceux rencontrés, c'est 
effectivement à l'aube de la trentaine que la décision d'abandonner la carrière criminelle a été prise. Un ex-détenu résume bien le dilemme auquel doit faire face celui qui fête ses trente ans en prison :

Rendu à 30 ans si un gars décide pas de prendre une autre direction, il va passer sa vie en dedans. Trente ans, c'est l'âge critique car à $\mathbf{4 0}$ ans tu peux pu te trouver de job, donc tu continues à voler.

Nous avons donc tenté de dresser dans les pages précédentes un tableau de ce que pourraient être certains aspects de la vie d'un détenu en prison. Ce tableau est fort sombre, il est même dans certains cas noir, nous en convenons. C'est une des dimensions de la vie en prison qu'il ne nous faut pas négliger, lorsqu'on tente de comprendre le mécanisme de l'abandon de la carrière criminelle. Les diverses expériences, que nous avons décrites, et beaucoup d'autres contribuent à amener le détenu à un point de saturation où il doit faire un choix. Par ailleurs, ce ne sont pas ces seules expériences qui comptent.

Quels autres facteurs rentrent en ligne de compte à ce moment-là de leur vie, facteurs qui les poussent en avant dans une nouvelle vie? C'est ce que nous allons tenter de décrire dans les pages suivantes.

\section{B. FACTEURS INCITANT $\AA$ UNE VIE NORMALE}

Même si certains ex-détenus ont insisté, avec vigueur, sur le fait qu'eux seuls ont décidé d'arrêter et que l'aide qu'ils ont reçue était secondaire, accessoire, insignifiante et, à la limite, inexistante, il n'en demeure pas moins, que certains d'entre eux ont effectivement bénéficié d'une quelconque forme d'aide, appui qui n'était pas, nécessairement, institutionnel, mais qui a concouru à leur faciliter la tâche de prendre et de maintenir leur décision d'abandonner.

Ainsi, certaines expériences, certaines découvertes intéressantes faites en prison peuvent quelquefois inciter le détenu à envisager l'hypothèse d'une nouvelle vie, ou encore affermir sa décision, et ainsi contribuer de façon positive à l'abandon de la carrière criminelle. Parmi celles-ci, il y a la découverte du savoir ainsi que le constat que l'on est capable de prendre des responsabilités. Dans son processus de prise de décision, le détenu peut 
être aidé et guidé par des organismes ou des individus périphériques à la prison, tels les Alcooliques Anonymes ou les aumôniers de prison. Enfin, la présence d'une épouse ou amie aimante et compréhensive, pendant ou après l'incarcération peut constituer un encouragement à persévérer dans la voie de l'abandon.

Nous pouvons donc distinguer trois catégories d'aide dont bénéficie le détenu qui désire, selon l'expression, «accrocher les gants». Ces catégories correspondent d'ailleurs à trois niveaux de relations entre individus. L'aide peut être officielle et ainsi provenir du milieu institutionnel et ceci dans une perspective de réhabilitation ou de resocialisation. Elle peut être périphérique au milieu (A.A., aumôniers), c'est souvent une aide morale au sens large. Enfin, elle peut être d'ordre plus sentimentale comme c'est le cas du soutien apporté par les épouses ou ami (e) $s$ de cœur. Nous allons nous y intéresser tout à tour.

\section{a) L'AIDE ISSUE DU MILIEU INSTITUTIONNEL}

\section{1) La découverte de nouvelles connaissances}

Parmi les principaux types d'aide dont bénéficie l'incarcéré, il y a la possibilité d'apprendre un métier ou de s'instruire, voire dans certains cas marginaux, de s'alphabétiser. Puisque dans certains cas - et ils ne sont pas en majorité - , le détenu peut être ignare. Il faut donc commencer au bas de l'échelle, lui apprendre à lire et à écrire, car il sait compter «quand on a à se séparer le butin », nous soulignait avec une pointe d'humour, un de nos répondants.

En plus des cours de niveau primaire, secondaire et postsecondaire dispensés dans les pénitenciers et qui permettent aux détenus d'améliorer leurs connaissances générales, le pénitencier donne également des cours d'apprentissage d'un métier et d'autres cours plus spécialisés visant à introduire le détenu sur le marché du travail à sa sortie de prison et ainsi faciliter sa réinsertion socioprofessionnelle.

Ainsi, c'est souvent en prison qu'un détenu va acquérir, pour la première fois de sa vie, les rudiments d'un métier, et c'est souvent pour lui la découverte d'un potentiel, qu'il ignorait posséder. Ainsi l'un d'eux, grâce à un psychologue, découvre qu'il est habile de ses mains. Il apprend qu'elles peuvent lui servir à autre chose qu'à tenir un revolver pour gagner sa vie. Il entreprend, alors, des cours en ébénisterie. Aujourd'hui, il peut, grâce à 
son apprentissage et à sa dextérité, gagner confortablement sa vie dans ce domaine, et il est, de plus, à son propre compte, ce qui n'est pas à négliger comme avantage pour un ex-détenu, qui tient jalousement à conserver le maximum de liberté de mouvement.

D'autres prendront des cours de plomberie, de mécanique, d'électronique, de programmation, selon leurs goûts et leurs aptitudes, et selon évidemment les disponibilités offertes par le milieu carcéral. Quelques-uns, souvent ceux qui ont de longues peines, vont mettre plusieurs cordes à leur arc en se familiarisant avec plusieurs métiers. Ils font d'une pierre plusieurs coups : car ce qui leur permet de briser la monotonie de la vie carcérale, pendant leur séjour en institution, contribuera à les rendre plus polyvalents sur le marché du travail. C'est le cas d'un multirécidiviste qui a suivi des cours de coiffure, de peinture, de dessin industriel et d'informatique; il a été longtemps et à plusieurs reprises pensionnaire des institutions carcérales. Mais prendre des cours et obtenir des diplômes en prison n'est pas un gage automatique de succès, pour se trouver un emploi, tout comme c'est le cas dans le monde extérieur. En effet, il est le seul ex-détenu, de tous ceux que nous avons rencontrés, qui était à ce moment-là sur l'Aide sociale.

Il arrive que des détenus, déjà en partie scolarisés, entreprennent des études conduisant à un métier. Quelquefois, le fait d'apprendre un métier peut conduire à autre chose; le détenu découvre que «ce n'est pas pour lui» comme on nous l'a dit, il réalise alors qu'il désire faire autre chose. Il décide, quelquefois, d'entreprendre des études plus poussées et certains poursuivront leurs études, mêmes après leur libération, dans certains cas jusqu'au niveau universitaire.

Dans d'autres situations, ce seront des études de perfectionnement d'un niveau plus technique, comme dans un cas qu'on libéra incidemment, plus tôt que prévu, pour lui permettre de commencer son année scolaire à temps, dans une école spécialisée en électronique. Aujourd'hui, il est propriétaire d'un commerce qui vend et répare des téléviseurs, et des jeux électroniques. C'est évidemment une entreprise fort prospère!

Également importants, sont les cours qui visent à développer chez le détenu de nouveaux centres d'intérêt tels les cours de peinture, de travail de cuir, et ces cours, au-delà du plaisir qu'ils procurent dans l'immédiat et dans l'avenir (plusieurs ex-détenus peignent encore dans leurs heures de loisir), peuvent quelquefois 
conduire à des résultats intéressants et quelquefois inespérés. C'est ainsi qu'un ex-détenu, qui a appris le travail du cuir en prison, par plaisir, est maintenant propriétaire d'une entreprise très rentable dans ce domaine et engage plusieurs personnes, dont certains sont incidemment d'anciens détenus. Et à sa sortie, un autre a vendu une toile exécutée pendant ses nombreuses années en prison, ce qui lui a permis de s'acheter une voiture, investissement indispensable pour lui, car il demeure à la campagne.

«... le plus important, c'est d'apprendre», voilà surtout ce que beaucoup d'ex-détenus ont découvert en prison. Chez certains, ce fut l'apprentissage d'un métier, chez d'autres cette trouvaille s'est traduite par une scolarisation plus poussée. L'étude peut contribuer, pour beaucoup, à la décision d'abandonner, et à la maintenir. Ainsi, quelqu'un qui a terminé son Cegep ${ }^{1}$ par correspondance au pénitencier nous avoue :

L'étude ça m'a assagi un peu, j'ai lu beaucoup, je prenais le maximum de chances (d'opportunités) que je pouvais prendre, telles les rencontres avec les gens de l'extérieur, j'ai lâché les gangs.

Et non seulement «ça assagi» mais ça peut également permettre de devenir conscient qu'on a d'autres possibilités en soi que celles de faire des hold-up, qu'on peut faire des choses dont on ne se serait jamais cru capable.

Un ex-voleur, qui a suivi des cours en arts graphiques de niveau post-secondaire en prison par l'intermédiaire d'un Cégep montréalais, en plus de ses cours de travail sur cuir, nous raconte une expérience qui l'a marqué profondément :

J'ai pris tous les cours que j'ai pu, j'avais de bonnes notes, ça me donnait de plus en plus confiance, surtout que j'étais au niveau collégial. Un jour, il y a eu un concours de graphisme. Il fallait faire un poster (sur un thème donné). J'ai gagné le concours, et mon poster a été imprimé. C'était plus important que de se faire payer.

La décision d'entreprendre des études est une décision importante dans la vie du détenu car elle marque un premier pas dans une nouvelle direction et, si elles sont poursuivies et réussies, au-delà de la satisfaction et de la confiance en soi qu'en retire

1. Collège d'enseignement général et professionnel. 
l'étudiant, elles indiquent une capacité de persévérer dans une tâche quelquefois ardue, attitude qui manquerait chez plusieurs criminels.

Un autre, qui songeait depuis longtemps à s'évader de prison, nous raconte :

... je me ramasse avec un total de huit ans et deux mois. J'ai trouvé ça pas drôle, et Archambault c'est difficile de s'évader de là. Je me suis alors souvenu que chaque fois que j'étais en dedans je faisais des études.

Un jour, je me rappelle même du ciel ce jour-là - il était chargé - je sens un changement en dedans de moi; il fallait que je fasse quelque chose : soit m'évader, soit faire des études et faire mon temps. J'ai décidé de faire mon temps et des études.

Et il fit des études au pénitencier, poursuivit au Cegep après sa libération, et fut admis à l'Université.

\section{2) La découverte de responsabilités}

La structure carcérale permet quelquefois aux détenus de se développer un sens des responsabilités et de réaliser des choses qu'ils ne se seraient jamais cru capable de faire. En somme, on se découvre des potentialités jusqu'alors latentes.

C'est ainsi que certains détenus vont assumer un certain nombre de responsabilités au sein de la vie carcérale. C'est le cas de l'un d'eux qui se retrouve en charge du comité des détenus, ce qui fait qu'il "est respecté en dedans et respecté en dehors», parce qu'il «marchait avec les deux bords : les gars et l'administration» et il retire avec raison une certaine satisfaction de se sentir respecté par les deux pôles du milieu carcéral. D'autres seront rédacteur du journal des détenus, représentant d'une "wing», organisateur pour les sports, les loisirs, des événements spéciaux, etc.

Lors de ces expériences gratifiantes en soi, ils seront appelés à établir des contacts avec l'extérieur, soit téléphoniques, soit beaucoup mieux encore, par des sorties pour vaquer à leurs occupations et assumer leurs nouvelles responsabilités. «Ça me fait des sorties, donc ils me font confiance, ils voient que tu peux agir en homme»; et, rajoute un ex-détenu, "parce que si tu as seulement des contacts avec l'intérieur, ça vaut pas la peine». 
Nous avons tracé, plus haut, un noir tableau de la vie en prison, mais tout n'est pas toujours si noir en prison et quelquefois on peut s'y amuser ferme, même si aux yeux du commun des mortels ces petits plaisirs paraissent puérils. Ainsi un ex-criminel, qui était en prison pour homicide et qui nous a avoué qu'il n'avait pas «un sens énorme des responsabilités», s'en découvre, tout en s'amusant à la prison, lorsqu'il organise des activités spéciales (vacances) pour les gens du pénitencier, pendant une quinzaine de l'été :

Un été i'ai organisé les vacances pour les gars. C'était au Centre fédéral. J'avais un budget de $1200 \$$ pour 400 gars, et pour 15 jours. J'ai réussi et il restait de l'argent après. J'ai téléphoné aux biscuits David pour avoir des bonbons, des choses comme cela, gratuitement. Le gars aux biscuits David m'a dit : «Gratuitement pour le pénitencier, et ben! Vous en avez du culot, on s'est fait défoncer hier soir!». Je lui ai répondu : "Je peux vous assurer que c'est personne de notre gang, on est pas sorteux, ils nous gardent en dedans.» Il a éclaté de rire et j'ai eu plus que ce que je lui demandais.

Il avait, également, négocié avec les autorités du pénitencier et le Club Playboy, la venue des «Bunnies» pour jouer à la balle molle avec les détenus. «Elles ont gagné 13 à 1 , il y avait toujours une «Bunny» qui courait d'un but à l'autre...»

Ainsi, tout en s'amusant, il s'est découvert un sens de l'organisation, qu'il ignorait posséder; il a ainsi développé un sens des responsabilités qu'il avouait ne pas posséder antérieurement. Suite à cette expérience d'organisation de "vacances», il "s'impliquera de plus en plus dans les activités du pénitencier». Aujourd'hui, il occupe un poste où il exerce des responsabilités importantes, et où il est appelé constamment à prendre des décisions qui impliquent la sécurité physique de plusieurs milliers d'enfants dans une grosse commission scolaire.

\section{b) L'AIDE PÉRIPHÉRIQUE AU MILIEU INSTITUTIONNEL}

Certaines formes d'aide qui font appel au bénévolat ou au quasi-bénévolat, sont souvent fort appréciées de certains détenus. C'est particulièrement le cas de l'aide fournie par les Alcooliques anonymes et celle fournie par des aumôniers, en prison.

\section{1) Les Alcooliques anonymes}

Les A.A., nous l'avons déjà souligné, sont très présents au sein des institutions carcérales et fournissent par leurs actions et 
les exemples qu'ils véhiculent, une aide considérable. Cette action est axée autour de conférences, de rencontres et de parrainage. En bref, elle s'organise de la façon suivante : lors d'un meeting, un conférencier, ex-alcoolique, vient raconter ses nombreux déboires avec l'alcool, tous les problèmes occasionnés par son éthylisme, et comment il s'en est finalement sorti. Ces histoires sont souvent effrayantes, on y raconte souvent avec l'humour noir particulier aux meetings A.A. - c'est une technique pour faire passer plus facilement le message - comment on s'est ruiné, comment on a perdu femme et enfants, comment on s'est retrouvé très bas, avec les robineux dans certains cas. Mais également, et c'est le côté positif du message, comment on s'en est sorti en abandonnant l'alcool et en adhérant à la philosophie des A.A. et à leur style de vie.

Pour le détenu le message peut être double. Pour le détenu alcoolique, ou ex-alcoolique, qui doit peut-être une partie de ses déboires avec la justice à une consommation abusive d'alcool, comme celui qui a tué et ne s'en rappelle pas, car il était alors «sur une cuite monumentale» - , le témoignage de ceux qui s'en sont tirés, qui ont recommencé à neuf en abandonnant l'alcool devient un exemple que l'on pourrait suivre. On sait que c'est possible, car beaucoup d'autres ont réussi et s'en trouvent maintenant mieux. Dans le cas de celui qui veut abandonner l'alcool, le parrainage par un ancien alcoolique qui s'en est sorti, vient ajouter une dimension plus personnelle et contribue à créer des liens d'amitié et de soutien, qui souvent se poursuivront après la libération.

Pour le détenu qui n'a pas, ou n'a pas eu de problèmes majeurs avec l'alcool, le témoignage humain a sa valeur intrinsèque :

Les A.A., c'est bien important en dedans. J'allais écouter cela.

Ça te marque d'entendre conter la vie des gens qui ont tout perdu, et qui s'en sont tirés.

En somme, comme le soulignait également un autre : «les A.A. c'est important car ça s'applique à n'importe quel problème de la vie. Ce sont des gars qui pensent positif, pas des gars qui parlent rien que de hold-up.»

\section{2) Les aumôniers de prison}

Dans nos sociétés modernes occidentales et rationalistes, la religion n'a plus l'importance qu'elle avait naguère. Il n'en reste 
pas moins que, chez certains détenus, elle fournit un réconfort moral et peut même être un facteur d'aide dans la décision d'abandonner, comme chez celui qui nous disait : «je priais tous les soirs, ça m'a pas fait de tort dans une prise de décision». Mais, il s'agit là vraiment de cas exceptionnels, tout comme chez cet autre, qui d'ailleurs associe sa croyance religieuse à la «mystique» des A.A. :

J'ai une grande croyance intérieure. Je côtoie beaucoup de religieux. Je fais quotidiennement la prière A.A. En dedans, j'étais régulièrement à la chapelle, je faisais même partie de la chorale. J'ai de la place dans ma vie pour du spirituel, du calme en masse. Je ne suis pas toujours dans le trafic.

Et il nous explique que cette découverte du spirituel, de ce qu'il appelle le "calme», lui a permis de s'en sortir, car il a découvert une dimension de lui-même qu'il ne connaissait pas. Enfin, un dernier nous avoue qu'il a vécu une phase mystique en prison, mais que sa décision d'abandonner répond à un autre critère que la religion : een dedans j'étais très croyant, je priais souvent, j’ai demandé à Dieu d'arrêter. Mais ma décision, c'est la peur».

Il arrive plus fréquemment que, l'aide apportée par la religion n'est pas à strictement parler d'ordre spirituel, mais plutôt une aide temporelle. C'est ainsi qu'un aumônier de prison, compréhensif et humain, peut devenir une personne ressource de valeur, un confident, un ami qui aide et qui conseille, et continue son ministère, perpétuant son aide, après la libération du détenu, comme le font d'ailleurs les A.A., ce qui souvent contribue à l'aider à persévérer dans la décision d'arrêter. Ainsi, un ex-détenu parmi d'autres, nous disait : «Quand ça va mal, $\mathrm{j}$ 'appelle le père $X$, et il me remonte le moral», et il n'est pas seul à faire appel à ce type d'aide dans les moments difficiles.

\section{LES ÉPOUSES OU AMIES}

L'épouse ou l'amie joue, quelquefois, un rôle important dans la décision d'abandonner la carrière criminelle, soit qu'elle ait été un support constant pendant ces longues années et a encouragé le détenu à revenir dans «le droit chemin», soit qu'en le quittant suite à ses nombreuses incarcérations ou à une dernière peine très longue, elle lui a fait prendre brusquement conscience de ce qu'il avait perdu par ses actes illégaux. Chez ces derniers abandonnés, la découverte ultérieure d'une nouvelle relstion avec une 
femme, soit en prison, soit à leur sortie a pu également affermir leur décision d'abandonner. Nous allons étudier quelques-unes de ces situations.

Ainsi, un multirécidiviste qui est parmi ceux qui ont maintenu de bonnes relations avec leurs épouses, pendant ses périodes d'incarcération, parce qu'elle l'a «attendu», et selon lui, elle aurait pu le faire pendant longtemps, - il avait commencé sa carrière criminelle dans la trentaine - ce qui est exceptionnel et il a maintenant 49 ans, nous disait :

Depuis l'âge de 17 ans je suis avec ma femme actuelle. Ma femme, c'est une one-man-woman elle dit : «il est assez écoeurant, j'en veux pas d'autre». Elle aurait attendu 25 ans pour moi. j'était pas malheureux en dedans, car je savais que quelqu'un m'attendait en dehors. Ma femme est gérante chez (grand magasin), elle pourrait me faire vivre.

Certains ex-détenus ont, par contre, connu de sérieuses difficultés avec leur épouse, suite à leurs problèmes avec la justice. Le témoignage suivant relate une pénible expérience dans le domaine des relations matrimoniales.

Au moment du sept ans, le gros problème c'était ma femme. Quand je rentrais dans ma cellule le soir, je me disais : «prépare-toi à perdre ce que tu aimes le plus». Je me préparais! Si elle ne m'aime pas, elle va me laisser aller. Elle ne me l'a jamais dit, n'en a jamais discuté avec moi, elle m'a laissé tomber. Je lui ai écrit, elle n'a jamais répondu à mes lettres. C'est pourquoi j'ai pris des procédures pour lui enlever les meubles, car elle n'a jamais répondu à mes lettres.

Ainsi, certains conservent l'amour de leur femme, malgré vents et marées, d'autres se retrouvent seuls en prison. Parmi ces derniers, lorsqu'ils décident finalement d'abandonner la carrière criminelle et de recommencer une nouvelle vie dans une autre direction, plusieurs envisagent sérieusement l'hypothèse d'entreprendre ce cheminement avec une compagne.

\section{a) LA PASSION ET LA RAISON}

Il y a plus, dans cette décision, que le fait de vouloir compenser l'absence quasi totale de relations hétérosexuelles endurée en prison et de vouloir maintenant reprendre le temps perdu dans ce domaine. En effet, chez beaucoup d'ex-détenus, parmi ceux que nous avons rencontrés, la femme représente plus que le simple 
«repos du guerrier», plus qu'une simple passion, elle peut dans certain cas incarner un facteur de stabilité, voir d'autorité.

Lorsque, selon ses dires, un de nos interviewés a «planifié» sa sortie, il a tenu compte des deux critères précédents. Il désirait un type de femme assez précis avec laquelle il pouvait avoir une relation sexuelle intense, ce qui correspondait à ses besoins dans le domaine, mais qui devait également lui imposer une certaine discipline. Elle devait être autoritaire, une «marâtre» comme il nous le dira d'ailleurs dans l'entrevue :

À ma sortie, j'avait tout planifié cela. Il me fallait une petite fille ben autoritaire, une petite blonde, autoritaire, qui aime jouer au cul, de préférence une Française. Je l'ai rencontré après avoir terminé mes cours à $(\mathrm{X})$. Elle m'a «dompé» un an après.

Un autre de son côté, sans mettre d'emphase sur le côté sexuel de ses relations avec sa femme, nous raconte une expérience qu'il venait de vivre récemment, et qui nous donne une idée de l'emprise qu'elle exerce sur lui en termes d'autorité :

Des fois en magasinant, je sortirais sans payer, elle ne veut rien savoir, elle est contre cela. La semaine dernière quand j'ai fait la commande d'épicerie, la caissière avait oublié de me faire payer. Quand ma femme l'a appris à la maison, elle m'a fait des reproches, et elle est allée payer la commande.

Une attitude similaire se retrouve dans le discours de beaucoup d'ex-détenus que nous avons rencontrés. La femme incarne l'autorité chez beaucoup d'entre-eux et leur en impose. Ainsi, l'un d'eux qui travaillait, après sa libération, à l'extérieur de Montréal, tout en fréquentant occasionnellement son ex-femme pendant les fins de semaine, se trouve du travail à Montréal. Il nous avoue : «à Montréal, j'achète des meubles cash, j'avais l'argent. Ma femme avait décidé qu'on resterait ensemble». Or, il a trempé dans toutes les combines possibles, il a même tué de sang-froid quelqu'un qui lui devait de l'argent et qui ne voulait pas le rembourser. Ce n'est donc pas un chérubin.

\section{b) LES RESPONSABILITÉS}

Plusieurs ex-détenus n'ont pas eu à assumer de responsabilités sérieuses depuis de nombreuses années. La vie en prison ne s'y prêtant guère. Si on fait exception des responsabilités, relativement marginales, exercées par quelques-uns en milieu carcéral, plusieurs 
en pratique n'ont jamais été en position de responsabilité pendant leur vie précarcérale et carcérale, ou y répugnaient carrément.

Le terme «responsabilité» est revenu souvent dans le discours de nos répondants. Il semble recouvrir deux réalités bien distinctes dans le cas qui nous intéresse ici, c'est-à-dire le fait de vivre avec une femme. Un premier sens peut être associé au fait que c'est la femme qui détient l'autorité dans le ménage, elle en est donc "responsable». Ainsi que nous le dit un de nos interviewés, dont l'épouse était propriétaire d'une entreprise :

Elle représentait la sécurité en dehors (de la prison) pour moi, elle était plus responsable, car elle était plus mature que moi.

Et cet autre, dont la femme «a décidé qu'ils vivraient ensemble», nous avouait également : «je n'ai pas eu de responsabilités depuis 16 ans, c'est bien dur d'en prendre, mais ma femme elle, elle est très solide».

Si certains s'appuient sur leur femme pour assumer les responsabilités du ménage, certains autres prennent la décision d'y faire face, ou encore de les partager. C'est souvent le cas d'exdétenus qui vivent avec une femme qui a déjà des enfants, ou à laquelle ils font des enfants. L'un d'eux, qui était atanné de changer de fille», a connu son épouse après sa libération :

Je voulais du travail en sortant. Je me suis placé assez facilement, mais j'avais besoin de quelqu'un à mes côtés, ... une femme! J'ai frappé plus : une femme avec des enfants. C'est surtout elle qui m'a incité à l'honnêteté.

Elle avait, alors, déjả trois enfants, il a agrandi la famille, de façon considérable, ils sont maintenant responsables de cinq enfants et ils ont monté une entreprise, dans le seul domaine qu'ils connaissent bien tous les deux, l'entretien-ménager. Un autre a également rencontré dans sa deuxième femme un support important : "quand j'ai rencontré ma femme on était deux épaves, on a trouvé un radeau, elle a été superbe, elle m'a aidé au bout».

\section{c) CAUSE DE L'ABANDON?}

Une femme peut, dans certaines circonstances, être plus qu'un soutien à l'abandon, elle peut en être la cause directe. Ce fut le cas d'un ex-criminel qui vécut une histoire d'amour, digne d'un roman. Au moment où il est incarcéré pour meurtre, son épouse 
est enceinte. Suivra le divorce et ils se perdent de vue pendant de nombreuses années. Il s'évade de prison et entre en communication avec ses anciens amis pour être dépanné et ces gens sont également les amis de son ex-épouse. Ils se rencontrent par hasard (?) et reprennent la vie commune. C'est à ce moment qu'il découvre sa petite fille, qu'il n'a jamais connue et qui a, alors, sept ans; et il réalise qu'il aime ce genre de vie. Il sera éventuellement arrêté lors d'une tentative de vol à main armée et réincarcéré. Mais, il a pris à ce moment-là une décision : celle de vivre avec son ex-femme et son enfant. Il s'en tirera, et il coule maintenant des jours heureux avec sa femme et ... ses trois enfants. En somme, s'évader de prison peut, à l'occasion, être un facteur qui contribue à l'abandon!

En somme, la femme représente un soutien notable à l'abandon quand elle n'en est pas la cause directe. Plusieurs auteurs avaient déjà relevé ce fait. Cette influence féminine aurait pu, semble-t-il, s'exercer même au début et servir d'antidote à l'entrée dans une carrière criminelle. Comme on nous le mentionnait :

J'avais jamais sorti avec une fille steady (régulièrement). Vers 18 ans, si j'avais eu une blonde, j'aurais été moins porté à me tenir avec les gars, et à me faire embarquer dans des coups.

Opinion qui semble confirmée par un autre : «J'ai été chanceux, j'avais ma femme (à ma sortie), mais les petits jeunes c'est différent, quand ils sortent, ils n'ont personne.»

Pour plusieurs ex-détenus, la fermme représente donc la passion et la raison. La passion car ils tombent ou retombent en amour et ne veulent ni blesser, ni perdre l'être cher. La raison au sens où la femme dans les ménages de certains ex-détenus incarne, dans une large mesure, la discipline, l'autorité, et est appelée à assumer une large part des responsabilités, ce sont des femmes fortes.

Mais laissons sur ce sujet le mot de la fin à un ancien du pénitencier :

Depuis 1973, ça va très bien mon affaire : j'ai toujours du travail. Je suis marié à une ancienne religieuse, elle dit que "vice et vertu s'adonnent". Je suis très bien avec ma femme et nos deux enfants, même si je me permets des petites vacances (amoureuses) de temps en temps. Mais dans la vie de couple ce que je n'aime pas, c'est qu'il y a un gardien et un prisonnier. 


\section{PERSÉVÉRER}

Se maintenir dans le «droit chemin» et ainsi conserver une liberté - enfin retrouvée - après plusieurs années de prison; est-ce difficile pour l'ex-détenu qui a décidé d'interrompre sa carrière criminelle? N'est-il pas quelquefois sujet à la tentation de reprendre les vieilles habitudes? A-t-il à faire face à des difficultés qui pourraient contribuer à le décourager et consécutivement à l'inciter à un retour au passé?

Soit parce qu'il se souvient des mauvaises expériences du passé, soit parce qu'il s'est découvert de nouvelles potentialités, de nouveaux intérêts, soit encore qu'il a bâti un ménage ou édifié une famille, qu'il possède d'autre part un emploi intéressant et rémunérateur, soit enfin qu'il évite les mauvaises fréquentations, voici autant d'atouts qui lui permettent de se bien tirer d'affaire et de persévérer.

Mais, en fait est-ce que les ex-détenus sont tous relativement bien immunisés contre les tentations? Certains nous ont affirmé qu'il ne leur était pas difficile de persévérer et cela, malgré de considérables difficultés matrimoniales ou financières. Mais, est-ce vrai pour tous?

\section{a) LES TENTATIONS}

Il semble que non, puisque quelques-uns nous ont avoué avoir eu des tentations depuis leur décision d'abandonner, et même d'en avoir encore. Ainsi l'un de nos répondants, qui a purgé une dernière sentence très longue en prison et qui n'est plus très jeune. s'est fait faire des propositions dès sa sortie de prison et même plus tard. Il travaillait, alors, dans une taverne où plusieurs «coups» se planifiaient sous ses yeux :

Ça m'a tenté. Des gars venaient me voir. Mais j'étais pas dedans, même pour des gros "scores». Je ne prends pas de chance, c'est ma vie! Je pogne pas cinq ans (si je me fais prendre) mais la vie.

Et cet autre, dont l'épouse vient d'avoir un enfant, avoue avoir encore des tentations et cela l'inquiète car il ne se croit pas assez fort pour y résister. Rappelons que c'est lui dont l'épouse a été régler l'addition de la commande d'épicerie quand elle a constaté que la caissière avait «oublié» de faire payer son mari : 
C'est officiel que j'ai des tentations fortes. J'ai jamais été mis à l'épreuve, sauf à ma shop où j'ai des responsabilités. Mais si je frappe à une porte et il $\mathrm{y}$ a personne, et il $\mathrm{y}$ a de l'argent qui traîne, je ne sais pas ce qui va arriver.

Et enfin, celui-ci qui ne peut résister aux tentations, quoique maintenant père de famille et travaillant à son compte, il se contente de peu, en termes d'activités criminelles. Il est, maintenant, plus prudent et plus modeste qu'à l'époque où il dévalisait banques, caisses populaires et dont l'opus major constitue une opération contre la Brink's, qui lui rapporta de nombreuses années de prison :

Aujourd'hui je fais un peu de vol à l'étalage, sinon je pourrais me remettre à faire des hold-up. J'ai besoin de me défouler.

Il ne faudrait pas conclure de ce qui précède, que tous les ex-détenus sont soumis à des tentations qu'ils ne peuvent réprimer. Certains y sont exposés, comme beaucoup d'honnêtes gens d'ailleurs, d'autres ont adopté un nouveau mode de vie d'où est exclue toute forme de contact avec le monde criminel, à l'instar de ceux qui se sont isolés à la campagne ou encore qui évitent toute relation avec les amis d'antan.

Donc, une fois prise la décision d'abandonner une carrière criminelle, est-il vraiment difficile de persévérer dans cette voie nouvelle? Dans ce domaine, comme nous venons de le souligner, les opinions et expériences des ex-détenus divergent. Certains concèdent n'avoir eu que peu ou pas de difficultés à maintenir leur décision. D'autres avouent avoir eu des problèmes au début mais les choses se sont améliorées par la suite. D'autres enfin vivent encore aujourd'hui et revivent constamment des problèmes reliés à leur passé criminel.

\section{b) CEUX QUI N'ONT PAS DE DIFFICULTÉS}

Parmi les ex-détenus qui affirment ne pas avoir eu de difficultés à maintenir leur décision d'arrêter et à persévérer dans cette décision, ceux pour lesquels cela nous semble avoir été le plus facile sont ceux-là même qui ont assumé publiquement leur situa. tion d'ex-détenu. C'est le cas de l'un d'eux qui a figuré dans le film de l'O.N.F., «Une vie en prison», qui participe à des conférences et des panels sur les prisons, en tant qu'ex-détenu, et qui enseigne actuellement à des ex-détenus analphabètes : 
J'ai eu aucune difficulté, au contraire, ça m'a aidé d'avoir été un détenu. Comme je travaille pour le collectif, ca m'ouvre des portes. Les ex-détenus aiment bien mieux se faire enseigner par l'un des leurs.

À sa sortie de prison, un autre a terminé son cégep et s'est inscrit à l'École nationale de théâtre. Alors qu'il est étudiant, l'École fait une exception à son règlement interne et lui permet de jouer dans une pièce pour le grand public. C'est une pièce écrite par des détenus et interprétée par des détenus dans un grand théâtre de Montréal. Sa figuration dans cette pièce lui vaudra d'être connu non seulement comme un ex-détenu mais également comme un jeune comédien fort talentueux, alors qu'il est - ne l'oublions pas -, toujours étudiant, et sous une libération conditionnelle : «il m’arrivait quelque chose de bien important : le succès», nous a-t-il avoué. Les ex-détenus qui s'affichent ouvertement comme tels - et c'est également le cas de celui qui fait des conférences chez les A.A. - , deviennent des exemples de réussite pour les autres ex-détenus. À ce titre, ils peuvent difficilement se permettre une rechute. Mais ceux qui persévèrent dans leur décision d'abandonner ne sont pas tous des gens jouissant d'une certaine notoriété. Ainsi, un ex-détenu, qui est maintenant camionneur, nous disait qu'il se sent beaucoup mieux maintenant qu'il a une vie rangée. Alors que nous lui demandions s'il trouvait cela dur de persévérer, il nous a rétorqué :

Je ne trouve pas ça dur. Au contraire, je suis heureux depuis ce temps-là. Il y des moments bas et des moments hauts, la vie est dure pour tout le monde. J'ai jamais eu la tentation de recommencer. Des fois j'ai le goût d'en assommer un, mais si tu te fais arrêter...? Ma femme et mon petit m'ont bien aidé à maintenir ma décision.

Si ce dernier possède un style de vie sédentaire, qu'il partage entre son travail, sa femme, son jeune fils et la modeste maison qu'il vient d'acheter et qu'il rénove dans ses moments de loisir, il n'en va pas de même pour cet autre qui est célibataire, qui voyage beaucoup, et qui court allègrement le jupon. Pourtant il n'a aucune intention de recommencer parce «le monde ne te demande pas mieux que de te donner leur argent, pourquoi leur voler?» Il faut dire qu'il est dans le commerce et que ses affaires vont très bien. Tout comme cet autre qui nous avouait :

Aujourd'hui, je sais que je peux faire beaucoup d'argent à pas voler. Pour moi, c'est le plus important. Aujourd'hui c'est 
tellement facile de faire de l'argent, pourquoi je prendrais des moyens illégaux.

Mais quand il ajoute «... si jamais je ne suis pu capable de faire de l'argent peut-être que je recommencerai», il nous signale un problème important auquel font face de nombreux ex-détenus à leur sortie de prison : «faire de l'argent». Pour le moment, lui n'a pas de problème à persévérer puisqu'il est financièrement à l'aise. Mais si les difficultés financières s'amoncelaient, si son entreprise faisait faillite? Comment réagirait-il?

\section{c) CEUX QUI AVOUENT CERTAINES DIFFICULTÉS}

Or, tous les ex-détenus ne se retrouvent pas dans une situation financière aussi enviable, et cela même quelques années après leur sortie de prison. Plusieurs trouvent cette situation très pénible, malgré leur détermination à persévérer : «Je ne trouve pas ça dur de continuer à arrêter, mais je trouve ça dur du côté monétaire», nous avouait l'un d'eux dont la femme ne travaille pas et qui a une fille adolescente et un fils de trois ans.

Si certains retirent une certaine fierté, et pourquoi pas le dire, certains avantages financiers à afficher leur abandon, si d'autres réalisent qu'ils font maintenant beaucoup plus d'argent avec un métier honnête, d'autres se sont découverts une famille, au moment de leur abandon, et cela les aide à persévérer. Un de nos interlocuteurs est parti de rien : à sa sortie de prison tout ce qu'il sait faire c'est de l'entretien-ménager. Il connaît sa femme par correspondance; elle a à ce moment-là déjà plusieurs enfants, d'un mariage précédent. Ils se marient et il fait des ménages pour faire vivre sa nouvelle famille :

$\mathrm{Au}$ moment de mon abandon, c'était nécessaire de faire vivre ma famille. J'ai réalisé que c'était plus payant de travailler légalement que de voler des téléviseurs de $800 \$$ pour les revendre $200 \$$. Ça en prend beaucoup pour faire de l'argent.

Il a, depuis, édifié une entreprise avec son épouse. Il gagne maintenant beaucoup d'argent et a une famille qu'il adore et qu'il ne voudrait pas perdre. Il a donc en main deux atouts pour persévérer. Et comme son travail l'appelle à travailler la nuit ou les week-ends, alors que les édifices où sa propre compagnie procède à l'entretien ménager sont vides, il ajoute : «si je voulais voler, je n'aurais même pas besoin de défoncer, j'ai les clefs d'un peu partout». 
d) CEUX QUI ONT ENCORE BEAUCOUP DE DIFFICULTÉS

Beaucoup moins bien lotis sont ceux qui sont sans famille ou qui l'ont perdue, et qui sont sans travail ou sans travail stable. C'est, en outre, la situation que vit actuellement un des interviewés, qui est en instance de divorce de sa deuxième femme, dont il a eu un jeune fils, et qui ne travaille qu'occasionnellement, puisque dans son métier (il est chef-machiniste dans le domaine de la production cinématographique commerciale), il ne peut être que pigiste. Persévérer, malgré ces difficultés, à quelque chose de quasi héroïque pour quelqu'un qui s'était habitué à la grande vie.

Depuis que j'ai accroché les gants je trouve ça très dur. Je vis seul sur une ferme, c'est très tough. Je vis d'espoir, espoir que ma femme revienne. Quand j'ai pas de travail, je n'ai que $600 \$$ par mois d'assurance chômage. Il faut que je paie la voiture $380 \$$ par mois, le loyer, etc., je mange des beans. J'aimerais avoir de l'argent pour me payer la traite moi aussi, c'est pour cela que je préfêre vivre à la campagne, en ville je deviens trop agressif. Avant de m'embarquer sur quelque chose je préfère vivre à la campagne. Je ne suis pas tenté de recommencer; ils peuvent venir chercher le «char», la maison est payée pour l'année, je voyagerai en autobus. Ma sécurité est liée à la campagne.

Beaucoup, sans nécessairement faire face à ces mêmes difficultés, ont également senti le besoin de s'isoler de la Métropole, entre autres choses pour éviter des rencontres fortuites qui pourraient les inciter à la tentation. «Dans le centre-ville, c'est trop facile de réembarquer, tu rencontres les gars de l'ex-gang : Veux-tu faire une job payante? Veux-tu de la coke (cocaine)? J'ai pensé que pour mettre toutes les chances de mon bord, c'était préférable d'éviter Montréal», nous disait l'un d'eux. Et pourtant il sortait dans des conditions optimales pour persévérer, il avait connu sa femme en prison (il restera avec elle cinq ans), et il avait un travail intéressant et rémunérateur. Les tentations devaient être vraiment fortes. Mais il a quand même persévéré jusqu'à ce jour.

\section{e) DIFFICULTÉS RELIÉES AU MARCHÉ DU TRAVAIL}

$\mathrm{Au}$ nombre des problèmes qu'affrontent les ex-détenus et qui peuvent contribuer à miner leur décision de persévérer, outre les tentations, il y a le fait de se retrouver en difficulté sur le marché du travail. Car, en effet, interrogés sur le type d'aide dont ils auraient eu le plus besoin, nos ex-détenus ont mis l'accent sur 
deux points : il faut être décidé d'une part et d'autre part avoir du travail en sortant. Ainsi, plusieurs ont encore souligné le fait qu'il faut avoir pris la décision d'abandonner sinon quelle que soit l'aide apportée, elle risque d'être inutile, inefficace : «il faut que le gars veule s'en sortir au départ», nous mentionnait-on, il faut que «les gars se sortent le petit peu de cour qu'ils ont dans le corps», car, rajoute-t-on, «il y en a beaucoup qui ont été aidés et qui sont encore en dedans».

Avoir du travail en sortant, c'est l'aide la plus importante qui, selon eux, peut leur être accordée. Mais obtenir du travail en période de chômage, et quand on est un ex-détenu, ce n'est sûrement pas facile: il faut donc surmonter un certain nombre de problèmes.

Ainsi, un ex-détenu considère que l'opinion publique est contre le fait que des ex-détenus obtiennent des emplois en sortant, alors qu'il y a tant d'honnêtes gens en chômage qui veulent travailler : «l'opinion publique dit : «moi je veux travailler, l'autre qu'il retourne en dedans». Et, un autre ajoute que des organismes comme les Centres de main-d'oeuvre, quand ils ont trouvé un travail à un ex-détenu, vérifient s'il est effectivement au travail, ce qui met la puce à l'oreille du patron qui ignore que son nouvel employé a un casier judiciaire. Dans certains types d'emplois, la situation est pire, selon lui, car ce sont les compagnies d'assurances qui vérifient «si tu es sécuritaire», ce qui peut conduire à la perte de l'emploi et à des conséquences fâcheuses :

Quand le détenu sort dehors, il faut lui montrer qu'on peut s'occuper de lui trouver un emploi, car il y en a qui se découragent s'ils sont refusés à cause de leur dossier.

Outre contribuer à vaincre la crainte qu'ont les employeurs face à un candidat qui possède un dossier, il y a le problème de la reconnaissance du nombre d'heures d'apprentissage exécutées en prison. On sait qu'un détenu peut avoir effectué un nombre impressionnant d'heures de travail à l'intérieur des murs dans un métier donné, cependant, ces heures ne sont pas reconnues, ce qui l'empêche d'obtenir sa carte de compétence dans son domaine, le mettant, ainsi, dans une situation défavorable sur le marché du travail : il est compétent mais sous-payé. Plusieurs ex-détenus ont souligné qu'ils ont eu à faire face à ce problème à leur sortie et qu'il faudrait y trouver une solution, afin d'aider vraiment celui qui sort plein de bonnes intentions pour l'avenir. 
On met également l'accent sur un problème auquel fait face toute personne qui a été absente du marché du travail pendant une longue période :

Il faut une période pour que le gars se retrouve lui-même, en dehors. Parce que du côté travail un gars est tout "dé-timé». Le patron devrait permettre aux gars de faire moins de huit heures par jour, pendant les premiers six mois. Un gars peut être plein de bonne volonté, mais il s'essouffle vite.

Un retour progressif au travail! Cette politique existe dans certaines entreprises publiques et privées, suite surtout à de longs congés de maladie, mais est-ce vraiment réaliste d'envisager un programme semblable dans le cas d'ex-détenus, surtout que souvent l'employeur est conscient qu'il prend déjà un risque? Quoi qu'il en soit, le problème est réel : un retour brutal sur le marché du travail est sûrement «essoufflant» pour quelqu'un habitué depuis des années à un rythme de vie nettement plus lent et moins stressant. Et si l'employé «s'essouffle (trop) vite», il risque de s'absenter souvent de son travail, ce qui peut devenir une cause de renvoi. Si l'ex-détenu perd son emploi et se décourage quelque temps après sa libération, que fera-t-il?

\section{f) «QUAND UN GARS VEUT, IL PEUT!»}

Alors est-il vraiment si difficile de persévérer? Pour certains, comme nous venons de le voir, cela ne semble pas constituer une difficulté majeure à surmonter, pour d'autre, cependant, l'accumulation des problèmes, une fois à l'air libre, pourrait les inciter à retourner sur les chemins du crime.

Ces difficultés peuvent être, dans une large mesure, reliées au marché du travail et à de faibles revenus, comme elles peuvent être issues d'une situation matrimoniale décevante, instable, sinon inexistante. Mais, si on tient compte du fait que beaucoup d'hommes, quel que soit leur passé, ont eu, un jour ou l'autre dans leur vie, à surmonter des obstacles similaires, et ne se retrouvent pas pour autant dans l'illégalité, pourquoi, à ce moment-là, les excriminels seraient-ils plus enclins à chercher des solutions à leurs problèmes financiers par des moyens illégaux? Parce qu'ils ont déjà goûté au fruit défendu? Parce qu'ils ont une connaissance intime des techniques qui permettent de faire beaucoup d'argent rapidement? Parce qu'un voleur demeure toujours un voleur? Nous ne saurions répondre! 
Mais, puisqu'ils ne succombent pas tous aux tentations, qu'est-ce qui les retient? Dans certains cas, comme nous l'avons vu, ce sera leur épouse et famille, dans d'autres cas, ce sera la peur de retourner en prison, ou encore une combinaison de plusieurs facteurs dissuasifs. Chez d'autres, le fait de se retirer à la campagne. d'éviter ainsi les anciennes fréquentations établit une barricade qui leur permet de s'isoler des tentations.

Mais, en dernière analyse, quand on a décidé d'arrêter, quelles que soient les raisons qui ont incité à prendre une telle décision et à la maintenir, il n'en demeure pas moins que c'est la volonté, la ferme intention de ne plus recommencer qui l'emporte et qui prime.

On exprimait bien l'essentiel de cette idée quand on nous affirmait précédemment : «Quand un gars veut, il peut. C'est vouloir qui est difficile!»

\section{CONCLUSION}

Quand on se penche sur le phénomène de l'abandon de la carrière criminelle, une constante apparaît clairement. C'est le fait que le criminel, en voie de changement, procède, à un moment donné, à un bilan de ses activités criminelles passées et des conséquences qui y sont attachées. Il évalue également de façon plus ou moins réaliste, selon les individus et selon le moment, les avantages qu'il pourrait retirer d'une vie plus normale. Le criminel serait ainsi d'une part poussé par la crainte d'être réincarcéré et d'autre part tiré par les perspectives de gratifications reliées à une vie conventionnelle.

Donc, dans la décision d'abandonner des activités criminelles il y aurait, si l'on reprend l'image populaire, le bâton et la carotte : d'une part, la peur du châtiment et de l'autre, l'espoir d'une récompense. Nous avons, dans le présent article, considéré ces deux aspects du problème et cela à partir des dires de nos interviewés. En brossant ce tableau, nous devons cependant garder à l'esprit que chez certains, plus pessimistes peut-être, c'est la peur de revivre les mauvaises expériences du passé qui a dominé dans la prise de décision et que chez d'autres, c'est une vision plus optimiste de l'avenir qui a été la fusée d'envol qui a précipité leur choix. Comme c'est souvent le cas dans les décisions de la vie courante, chez plusieurs c'est une combinaison de facteurs éloi- 
gnant de la vie criminelle, alliés à un certain nombre d'éléments attirant vers une vie normale qui auraient fait pencher la balance en faveur de l'abandon.

Ainsi, parmi les facteurs qui inciteraient les criminels à quitter la voie de l'illégalité, il y aurait d'abord les nombreux déboires liés au style de vie criminelle, auxquels se grefferont éventuellement les mauvaises expériences reliées à la vie carcérale.

Ces premiers déboires seront souvent occasionnés par leurs propres amis criminels ou encore résulteront de conflits avec des bandes rivales. Dans un cas comme dans l'autre, ils sont également à la merci d'une dénonciation, car chez les uns comme chez les autres, il y a des iscariotes et des envieux. D'autre part, le joug du système de justice se fait de plus en plus lourd : la police les harcèle plus fréquemment, les «bons» avocats deviennent de plus en plus exigeants et, finalement trouvé coupable, les juges font quelquefois en sorte que pour le multirécidiviste, le bât fasse de plus en plus mal. D'autre part, ce qui était un jeu, au départ, est devenu de plus en plus stressant et de moins en moins amusant avec le temps. On prend progressivement conscience que, plus on commet de délits, plus le risque de se faire prendre s'accentue.

Suite au dernier délit, on s'est donc retrouvé encore «en dedans» et on subit ou revit, alors, un certain nombre de mauvaises expériences reliées à la vie carcérale. Que ce soit au niveau des conditions de vie en prison, au niveau des relations avec agents, gardiens ou les autres détenus, ou même encore avec l'extérieur; l'accumulation d'expériences désagréables va contribuer à «écceurer» certains criminels de leur style de vie. Certains ont alors réalisé qu'en abordant la trentaine, il leur fallait prendre une décision : c'est l'âge critique où on doit se brancher, si on ne veut pas continuer indéfiniment à payer les frais.

D'autre part, certaines expériences peuvent encourager le criminel à envisager l'hypothèse de modifier son style de vie et à s'intéresser aux bénéfices à retirer d'une vie rangée.

Pour ceux qui décident d'abandonner, le milieu carcéral offre différents types d'aide. Ces aides qui sont, selon de nombreux exdétenus, à prendre ou à laisser, peuvent entraîner : soit la découverte du savoir, soit le constat également important qu'on est capable d'assumer des responsabilités conventionnelles et qu'on peut en retirer une certaine satisfaction, si ce n'est une certaine fierté. Quelquefois, l'aide n'est pas à strictement parler institu- 
tionnelle, car elle s'inscrit à la périphérie du milieu carcéral. C'est ainsi que les Alcooliques anonymes et la religion peuvent apporter un support à la décision d'abandonner. La présence, d'autre part, dans la vie de l'ex-criminel, d'une épouse ou d'une amie de cour compréhensive et aimante, introduit un élément sentimental dans la prise de décision, dimension passionnelle qui n'est pas à négliger.

Le seul fait d'avoir pris la décision d'abandonner, même si cette décision a été longuement mûrie pendant des années de vie carcérale et est maintenant ancrée dans la volonté de l'ex-criminel, ne nivelle pas pour autant tous les problèmes auxquels aura à faire face l'ex-détenu suite à sa libération. Le retour à la vie civile occasionne, en effet, des difficultés d'intensités différentes selon les individus. Certains en connaîtront très peu, d'autres auront à en surmonter de considérables. C'est dans une large mesure sur le marché du travail, ainsi que dans le domaine de ses relations matrimoniales et amicales que l'ex-détenu affrontera sa nouvelle vie.

Une situation agréable sur ces fronts facilitera et renforcera sa décision d'abandonner et de persévérer dans une nouvelle voie. En effet, être en amour, fonder une famille, avoir un travail intéressant et rémunérateur, sont des constantes qui aident l'ex-détenu à retrouver une vie normale.

Enfin, pour les moins chanceux qui affrontent des difficultés dans ces domaines, la peur de se retrouver en prison peut servir d'antidote, d'élément dissuasif, en présence des tentations incitant à retourner à l'ancien style de vie criminelle, et cela dans l'attente et l'espoir de temps meilleurs.

Quoi qu'il en soit, quel que soit le cheminement qui a finalement amené l'abandon, ce serait, selon les nombreux ex-détenus que nous avons rencontrés, surtout la «volonté» de ne plus recommencer qui l'emporterait. Comme l'exprimait si bien et si simplement l'un d'eux : \&Quand un gars veut, il peut, c'est vouloir s'en sortir qui est difficile.»

\section{BIBLIOGRAPHIE}

CONKLIN, J.E. (1981), Criminology, New York, MacMillan Publishing Co. Inc. 
CORMIER, B.M., M. KENNEDY, J. SANGOVICZ, M. TROTTIER, (1959), «The Natural History of Criminality and Some Tentative Hypotheses on Its Abatment», la Revue canadienne de criminologie, vol. 1, no 4, p. 35-49.

CORMIER, B.M. (1975), The Watcher and the Watched, Montréal, Tundra THIFFAULT, A. OBERT, (1965), «Criminal Process and Emotional Growth», in Cameron, E. (édit.), Forensic Psychiatry and Child Psychiatry, Boston, Little Brown and Co., p. 3-41.

CORMIER, B.M. (1975), The Watcher and the Watched, Montreal, Tundra Books.

CUSSON, M. (1983), le Contrôle social du crime, Paris, Presses universitaires de France, «Sociologies».

GLUECK, S. et Eleanor GLUECK, (1974), Of Delinquency and Crime, Springfield, Charles $\mathrm{C}$. Thomas.

IRWIN, J. (1970), The Felon, Englewoods Cliffs, N.J., Prentice Hall Inc.

MEISENHELDER, T. (1977), «An Exploratory Study of Exiting from Criminal Careers», Criminology, vol. 15, no 3, novembre 1977, p. 319-334.

PINSONNEAULT, P. (1984), l'Abandon de la carrière criminelle, Université de Montréal, École de criminologie, (Les Cahiers de l'École de criminologie, $n^{\circ} 15$ ).

SOLLICITEUR GÉNÉRAL DU CANADA (1981), Rapport annuel 19801981, Ottawa, Canada

WALLER, I. (1974), Men Released from Prison, Toronto, University of Toronto Press.

WEST, W.G. (1978), «The Short Term Careers of Serious Thieves», Revue canadienne de criminologie, 20 (2), p. 169-190. 